\title{
Structural Features and Phylogenetic Implications of 11 New Mitogenomes of Typhlocybinae (Hemiptera: Cicadellidae)
}

\author{
Shuanghu Lin $(\mathbb{B}$, Min Huang and Yalin Zhang * (1) \\ Key Laboratory of Plant Protection Resources and Pest Management, Ministry of Education, \\ Entomological Museum, College of Plant Protection, Northwest A\&F University, Yangling 712100, China; \\ bosstigers@163.com (S.L.); huangmin@nwsuaf.edu.cn (M.H.) \\ * Correspondence: yalinzh@nwsuaf.edu.cn; Tel.: +86-029-87092190
}

Citation: Lin, S.; Huang, M.; Zhang,

Y. Structural Features and

Phylogenetic Implications of $11 \mathrm{New}$ Mitogenomes of Typhlocybinae (Hemiptera: Cicadellidae). Insects 2021, 12, 678. https://doi.org/ $10.3390 /$ insects 12080678

Academic Editor: Giuliana Allegrucci

Received: 20 June 2021

Accepted: 26 July 2021

Published: 28 July 2021

Publisher's Note: MDPI stays neutral with regard to jurisdictional claims in published maps and institutional affiliations.

Copyright: (C) 2021 by the authors Licensee MDPI, Basel, Switzerland. This article is an open access article distributed under the terms and conditions of the Creative Commons Attribution (CC BY) license (https:// creativecommons.org/licenses/by/ $4.0 /)$.
Simple Summary: Typhlocybinae is the smallest sized and most evolved leafhopper, body length 2-4 mm, forewing with four apical cells but lacking closed preapical cells. It comprises over 6000 species (Dietrich, 2013) distributed worldwide, making it the second largest group of Cicadellidae. Previous phylogenetic analyses in this subfamily were mainly based on morphological characters and were restricted to several gene fragments. To provide further insight into the relationships of its included tribes, complete mitogenomes of two Alebrini species (Shaddai acuminatus, Sobrala sp.), two Dikraneurini species (Dikraneura (Dikraneura) zlata, Robusta emeiensis), two Empoascini species (Alebroides salici, Empoasca serrata), two Erythroneurini species (Elbelus tripunctatus, Kaukania anser), two Typhlocybini species (Eupteryx (Eupteryx) adspersa, Eurhadina jarrary), and one Zyginellini species (Yangisunda tiani) are newly sequenced and comparatively analyzed. The mitogenomes comprise the typical set of 37 mitochondrial genes and a large non-coding region (A+T-rich region). The acceptor arm of $t r n R$ is the most inconstant among all the tRNAs, due to the acceptor arm comprising unpaired bases. Phylogenetic analyses using Bayesian inference and maximum likelihood methods produced a well-resolved framework of Typhlocybinae, showed the monophyly of Typhlocybinae and its inner tribes, except for Typhlocybini and Zyginellini combined. These results provide the valuable data toward the future study of the phylogenetic relationships in this subfamily.

Abstract: To explore the characteristics of mitogenomes and discuss the phylogenetic relationships and molecular evolution of the six tribes within Typhlocybinae, 11 complete mitogenomes are newly sequenced and comparatively analyzed. In all of these complete mitogenomes, the number and order of the genes are highly conserved in overall organization. The PCGs initiate with ATN/TTG/GTG and terminate with TAA/TAG/T. Almost all tRNAs are folded into the typical clover-leaf secondary structure. The control region is always variable in length and in numbers of multiple tandem repeat units. The atp 8 and nad2 exhibits the highest evolution rate among all the PCGs. Phylogenetic analyses based on whole mitogenome sequences, with three different datasets, using both maximum likelihood and Bayesian methods, indicate the monophyly of Typhlocybinae and its inner tribes, respectively, except for Typhlocybini and Zyginellini that are paraphyletic. Finally, we confirm that Erythroneurini is a subtribe of Dikraneurini.

Keywords: Auchenorrhyncha; leafhoppers; phylogeny; tribes

\section{Introduction}

Members of Typhlocybinae are in the family Cicadellidae, Auchenorrhyncha, and order Hemiptera. They are distributed worldwide and their tropical fauna, in particular, is most diverse [1,2]. Typhlocybinae is composed of six tribes (Alebrini, Dikraneurini, Empoascini, Erythroneurini, Typhlocybini and Zyginellini) in the most well-accepted taxonomy system that was proposed by Dworakowska in 1979 [3]. The monophyly of Typhlocybinae has been strongly supported by previous studies [4-7]. Typhlocybinae 
are the smallest sized and one of the most evolved groups of leafhoppers, body length 2-4 mm and forewing with four apical cells but lacking closed preapical cells [1,4]. It is the second largest group of Cicadellidae with over 6000 species [8]. These leafhoppers are of great ecological and economic significance. Large populations of these species feed mainly on host plant leaf parenchyma cells, causing "hopperburn" to numerous crops including apple, grape, soybean and potato [1]. In addition, some serve as vectors of numerous pathogens and viruses to host plants. Previous investigations of Typhlocybinae have focused primarily on morphology-based taxonomy and limited gene fragments or incorporated only a few taxa into higher level phylogenetic studies [3-7,9]. Thus, the phylogenetic relationships among tribes of Typhlocybinae has not yet been adequately explored. Further investigation based on new characteristics, including mitochondrial genomes, is used urgently to reconstruct the phylogeny of Typhlocybinae.

The insect mitogenome is typically double-strand circular DNA molecules, containing 13 protein coding genes, 2 ribosomal RNA, 22 transfer RNA genes and a control region (A + T-rich region) of variable length and number of multiple tandem repeat units, that regulate transcription and replication [10-12]. The mitogenome provides genome-level information, including base composition, sequence arrangement, codon usage or variation, RNA secondary structures and control region characters. The mitogenome has unique features including matrilineal inheritance, intron deletion, low recombination and high evolutionary rates [12-14]. With the recent cost reduction of high-throughput sequencing, partial or complete mitogenomes are extensively used in population genetics, phylogeny, evolution and phylogeography investigations of insects during these past several years [11,15-22].

Previously, there were only 14 complete mitogenomes pertaining to four tribes (Empoascini, Erythroneurini, Typhlocybini and Zyginellini) of Typhlocybinae in GenBank (https:/ / www.ncbi.nlm.nih.gov, accessed on 12 April 2021). However, mitogenomes of Alebrini and Dikraneurini species were still absent. In this study, we use three different datasets (PCG123, PCG123R, PCG12) and use both maximum likelihood (ML) and Bayesian inference (BI) methods to reconstruct their phylogenetic relationships and provide further insight into their taxonomic status. This is based on 11 newly sequenced and functional annotated complete mitogenomes of two Alebrini species (Shaddai sp., Sobrala sp.), two Dikraneurini species, (Dikraneura (Dikraneura) zlata, Dikraneurini sp.), two Empoascini species (Alebroides salicis, Empoasca serrata), two Erythroneurini species (Elbelus tripunctatus, Kaukania anser), two Typhlocybini species (Eupteryx (Eupteryx) adspersa, Eurhadina jarrary), and one Zyginellini species (Yangisunda tiani) along with 13 previously available mitogenomes in GenBank. The genomic size, base composition and skewness, sequence arrangements, codon usage or variation, evolutionary rate, genetic distance, ka/ks, start and stop codons, RNA secondary structures and control region characters are analyzed.

\section{Materials and Methods}

\subsection{Sample Preparation and Genomic DNA Extraction}

Collection information for the specimens sequenced in this study is shown in Table S1. All sample species are widely distributed in China and their status are stable in the taxonomy system that was proposed by Dworakowska in 1979 [3], all the specimens were adults captured alive and preserved in $100 \%$ ethanol and stored in a $-20^{\circ} \mathrm{C}$ freezer in the Entomological Museum of Northwest A\&F University (NWAFU) until identification and DNA extraction. Specimens were identified to species according to their wing venations and male genitalia characteristics. Genomic DNA was extracted from the whole body of adult specimens using the EasyPureR Genomic DNA Kit following the manufacturer's protocol (TransGen, Beijing, China).

\subsection{Sequence Assembly, Annotation and Bioinformatic Analysis}

Complete mitogenomes of all 11 species were sequenced using next-generation sequencing at the Illumina HiSeq 2500 platform with paired reads of $2 \times 150 \mathrm{bp}$ by the 
Biomarker Technologies Corporation (Beijing, China), except for Shaddai sp. and Sobrala $\mathrm{sp}$. that were sequenced by Novogene Corporation (Beijing, China). The raw pairedend clean reads were quality-trimmed and assembled by Geneious 8.1.3 (Biomatters, Auckland, New Zealand) with default parameters, Empoasca onukii (MG190360), Empoascanara dwalata (MT350235) and Limassolla lingchuanensis (NC_046037) were utilized as references. All 11 complete mitogenomes are annotated in Geneious 8.1.3 (Biomatters, Auckland, New Zealand) [23]. 13 PCGs were predicted as open reading frames (ORFs), employing the invertebrate mitochondrial genetic code. Two rRNA genes and control regions were identified by the boundary of their adjacent genes and based on comparison with homologous genes from other Typhlocybinae species. The locations and secondary structures of 22 tRNA genes were predicted by the MITOS Web Server (http:/ / mitos2.bioinf.uni-leipzig.de/index.py, accessed on 12 April 2021) [24]; their secondary structures were then plotted manually using Adobe Illustrator CC2018 according to the predictions. The control region (A+T-rich region) tandem repeats were identified by the tandem repeats finder online server (http:/ / tandem.bu.edu/trf/trf.html, accessed on 12 April 2021) [25]. The mitogenome maps were produced by the CGView Server (http:/ / stothard.afns.ualberta.ca/cgview_server/, accessed on 12 April 2021) [26]. Analyses of the 11 complete mitogenomes, including nucleotide composition, composition skew, and relative synonymous codon usage (RSCU) were analyzed with PhyloSuite v1.2.2 [27]. Strand asymmetry was computed according to the formulas AT-skew $=(\mathrm{A}-\mathrm{T}) /(\mathrm{A}+\mathrm{T})$ and GC-skew $=(G-C) /(G+C)$ [28]. To detect the nucleotide diversity (Pi) of 13 PCGs among 22 complete mitogenomes of Typhlocybinae, a sliding window analysis using $200 \mathrm{bp}$ and a step size of $20 \mathrm{bp}$ were conducted by the DnaSP v6 [29]. The ratios of non-synonymous (Ka) and synonymous (Ks) substitutions rates for each PCG of 22 Typhlocybinae species were also computed using DnaSP v6. Genetic distances between 22 Typhlocybinae species based on each PCG were computed using MEGA X with Kimura-2-parameter [30].

\subsection{Sequence Alignment and Phylogenetic Analysis}

One Evacanthinae species (Evacanthus heimianus) [31] and one Coelidiinae species (Taharana fasciana) [32] were available and chosen as outgroups. A total of 22 complete mitogenomes (including 11 newly-sequenced and 11 available mitogenomes [33-41]) of Typhlocybinae species (including 2 Alebrini species, 4 Empoascini species, 2 Dikraneurini species, 5 Erythroneurini species, 5 Typhlocybini species and 4 Zyginellini species) were selected to perform the phylogenetic analysis (Table 1). All available mitogenomes in this study were obtained from GenBank.

PCGs, rRNAs and tRNAs were extracted in PhyloSuite v 1.2.2. PCGs were aligned using the MAFFT v 7.313 plugin in PhyloSuite v 1.2.2, rRNAs were aligned using MAFFT version 7 online server (https:/ / mafft.cbrc.jp/alignment/server/) (accessed on 12 April 2021) with the Q-INS-i strategy [42] Gblocks v 0.91b [43] plugin in PhyloSuite v 1.2.2 was used to remove gaps and ambiguous sites. Aligned PCGs and rRNAs were concatenated using PhyloSuite v 1.2.2, respectively. 
Table 1. Classification and origins of the mitogenomic sequences used in this study.

\begin{tabular}{|c|c|c|c|c|c|}
\hline Family & Subfamily & Tribe & Species & Accession Number & Reference \\
\hline \multirow{15}{*}{ Cicadellidae } & Evacanthinae & & $\begin{array}{l}\text { Evacanthus } \\
\text { heimianus }\end{array}$ & MG813486 & [31] \\
\hline & Coelidiinae & & Taharana fasciana & NC_036015 & [32] \\
\hline & \multirow{13}{*}{ Typhlocybinae } & Alebrini & $\begin{array}{l}\text { Shaddai sp. } \\
\text { Sobrala sp. }\end{array}$ & $\begin{array}{l}\text { MZ014457 } \\
\text { MZ014458 }\end{array}$ & $\begin{array}{l}\text { This study } \\
\text { This study }\end{array}$ \\
\hline & & Empoascini & $\begin{array}{l}\text { Ghauriana sinensis } \\
\text { Alebroides salicis } \\
\text { Empoasca serrata } \\
\text { Empoasca onukii }\end{array}$ & $\begin{array}{c}\text { MN699874 } \\
\text { MZ014449 } \\
\text { MZ014453 } \\
\text { NC_037210 }\end{array}$ & $\begin{array}{c}\text { [33] } \\
\text { This study } \\
\text { This study } \\
{[34]}\end{array}$ \\
\hline & & Dikraneurini & $\begin{array}{c}\text { Dikraneura } \\
\text { (Dikraneura) zlata } \\
\text { Dikraneurini sp. }\end{array}$ & $\begin{array}{l}\text { MZ014450 } \\
\text { MZ014451 }\end{array}$ & $\begin{array}{l}\text { This study } \\
\text { This study }\end{array}$ \\
\hline & & \multirow{3}{*}{ Erythroneurini } & $\begin{array}{c}\text { Empoascanara } \\
\text { dwalata } \\
\text { Empoascanara sipra }\end{array}$ & $\begin{array}{l}\text { MT350235 } \\
\text { NC_048516 }\end{array}$ & $\begin{array}{l}{[35]} \\
{[36]}\end{array}$ \\
\hline & & & $\begin{array}{c}\text { Mitjaevia } \\
\text { protuberanta }\end{array}$ & NC_047465 & [37] \\
\hline & & & $\begin{array}{l}\text { Elbelus tripunctatus } \\
\text { Kaukania anser }\end{array}$ & $\begin{array}{l}\text { MZ014452 } \\
\text { MZ014456 }\end{array}$ & $\begin{array}{l}\text { This study } \\
\text { This study }\end{array}$ \\
\hline & & \multirow{3}{*}{ Zyginellini } & Zyginella minuta & MT488436 & [38] \\
\hline & & & $\begin{array}{c}\text { Limassolla } \\
\text { lingchuanensis }\end{array}$ & NC_046037 & [39] \\
\hline & & & $\begin{array}{c}\text { Parathailocyba orla } \\
\text { Yangisunda tiani }\end{array}$ & $\begin{array}{l}\text { MN894531 } \\
\text { MZ014459 }\end{array}$ & $\begin{array}{l}{[40]} \\
\text { This study }\end{array}$ \\
\hline & & \multirow{4}{*}{ Typhlocybini } & $\begin{array}{l}\text { Bolanusoides } \\
\text { shaanxiensis }\end{array}$ & MN661136 & Unpublished \\
\hline & & & Typhlocyba sp. & KY039138 & [41] \\
\hline & & & $\begin{array}{c}\text { Eupteryx (Eupteryx) } \\
\text { adspersa }\end{array}$ & MZ014454 & This study \\
\hline & & & $\begin{array}{l}\text { Eupteryx minuscula } \\
\text { Eurhadina jarrary }\end{array}$ & $\begin{array}{l}\text { MN910279 } \\
\text { MZ014455 }\end{array}$ & $\begin{array}{c}\text { Unpublished } \\
\text { This study }\end{array}$ \\
\hline
\end{tabular}

Phylogenetic relationships were reconstructed by the maximum likelihood (ML) and the Bayesian inference (BI) methods based on three datasets: (1) the PCG123 matrix (comprising all codon positions of the 13 PCGs, 10,860 bp in total); (2) the PCG123R matrix (comprising all codon positions of the 13 PCGs and 2 rRNA, 12,998 bp in total); (3) the PCG12 matrix (comprising the first and second codon positions of the 13 PCGs, 7240 bp in total). The best partitioning schemes and evolution models of both ML analyses and BI analyses were inferred using PartitionFinder v 2.1.1 plugin in PhyloSuite v 1.2.2 using the greedy search algorithm with branch lengths linked and Bayesian information criterion (BIC) [44]. The optimal substitution results are shown in Tables S2 and S3. ML analyses were conducted using IQ-TREE v 1.6.8 plugin in PhyloSuite v 1.2.2 under ultrafast bootstraps with 1000 replicates [45]. BI analyses were conducted using MrBayes v 3.2.6, executed in the CIPRES Science Gateway (www.phylo.org) (accessed on 13 April 2021), with default settings and $5 \times 106$ million Markov chain Monte Carlo (MCMC) generations and sampling every 1000 generations [46]. When the average standard deviation of split frequencies fell below 0.01, it was considered to reach stationarity. The initial $25 \%$ of sampled data were cast off as burn-in, and the remaining trees were applied to generate a consensus tree and compute the posterior probabilities (PP). 


\section{Results and Discussion}

\subsection{Mitogenome Structure and Nucleotide Composition}

Structures and lengths of the 11 newly sequenced complete and circularized mitogenomes of Typhlocybinae in this study are shown in Figures 1 and S1. The length of mitogenomes are range from 15,131 to $17,575 \mathrm{bp}$, the length difference mainly due to the variable length of the A+T-rich region [47]. The number and order of the genes are highly conserved in overall organization, containing 13 PCGs, 22 tRNAs, 2 rRNAs and a control region. Among the 37 mitochondrial genes, 9 PCGs (nad2, cox1, cox2, atp8, atp6, cox3, nad3, $n a d 6, c y t b)$ and 14 tRNAs (trnI, trnM, trnW, trnL2, trnK, $\operatorname{trn} D, \operatorname{trn} G, \operatorname{trn} A, \operatorname{trnR}, \operatorname{trn} N, \operatorname{trnS1}$, $\operatorname{trn} E, \operatorname{trn} T, \operatorname{trnS2}$ ) are transcribed from the majority strand (J-strand), while the remaining genes, 4 PCGs (nad5, nad4, nad4L, nad1), 8 tRNAs ( $\operatorname{trn} Q, \operatorname{trn} C, \operatorname{trn} Y, \operatorname{trnF}, \operatorname{trnH}, \operatorname{trn} P, \operatorname{trnL1}$, $t r n V)$ and 2 rRNAs $(r r n L, r r n S)$ are encoded on the minority strand (N-strand).

The 11 mitogenomes present a heavy AT nucleotide bias, with AT\% across the whole sequence attaining $74.8-78.4 \%$, similar to other previously sequenced mitogenomes of Typhlocybinae species [33-41]. The composition skew analysis shows that all 11 mitogenomes present a positive AT-skew and a negative GC-skew in the whole mitogenome. However, in tRNAs, rRNAs and PCGs with its 1st codon and 2nd codon position, it presents an opposite result. In PCGs, the 2nd codon position has the lowest AT content, while the 3rd codon position has the highest AT content. The AT content of tRNAs is lower than in rRNAs. The 11 control regions show a variable AT content, from 59.3\% to 93.8\% (Table S4).

\subsection{Protein-Coding Genes and Codon Usage}

The total lengths of the 13 PCGs among the 11 complete mitogenomes range from 10,854 (Shaddai sp.) to 10,962 bp (Dikraneurini sp.). The atp 8 is the smallest gene while the nad5 is the largest gene. The total 13 PCGs also present a heavy AT nucleotide bias, with $\mathrm{AT} \%$ ranging from $74.1 \%$ to $76.5 \%$, but with a negative AT-skew and a positive GC-skew. Most of the PCGs start with ATN, but atp8, nad5 and cox2 start with TTG that occurred in some mitogenomes within the 11 species. GTG was found to be the initiating codon of cox2 in Empoasca serrata. The latter two initiating codons were also found in the previously sequenced mitogenomes of Cicadellidae. The PCGs always terminated with TAA, while the incomplete stop codon T appeared 33 times among all PCGs of the 11 complete mitogenomes, TAG was used the least, only 15 times (Table S5). The incomplete stop codon T may be changed into TAA by post-transcriptional polyadenylation during the mRNA maturation process [48].

The relative synonymous codon usage (RSCU) of the 22 complete mitogenomes of Typhlocybinae used in this study, was computed and is presented in Figures 2, S2 and S3, showing a roughly similar RSCU among all 22 species. The most frequently used codons are AUU-Ile, UUA-Leu2, UUU-Phe and AUA-Met, all of which they are composed wholly of A and $\mathrm{U}$, indicating the codon usage has a heavy AT nucleotide bias, which explains the nucleotide AT bias in the PCGs among typhlocybine species. Additionally, the codon Arg (CGC) in Bolanusoides shaanxiensis was not observed. 

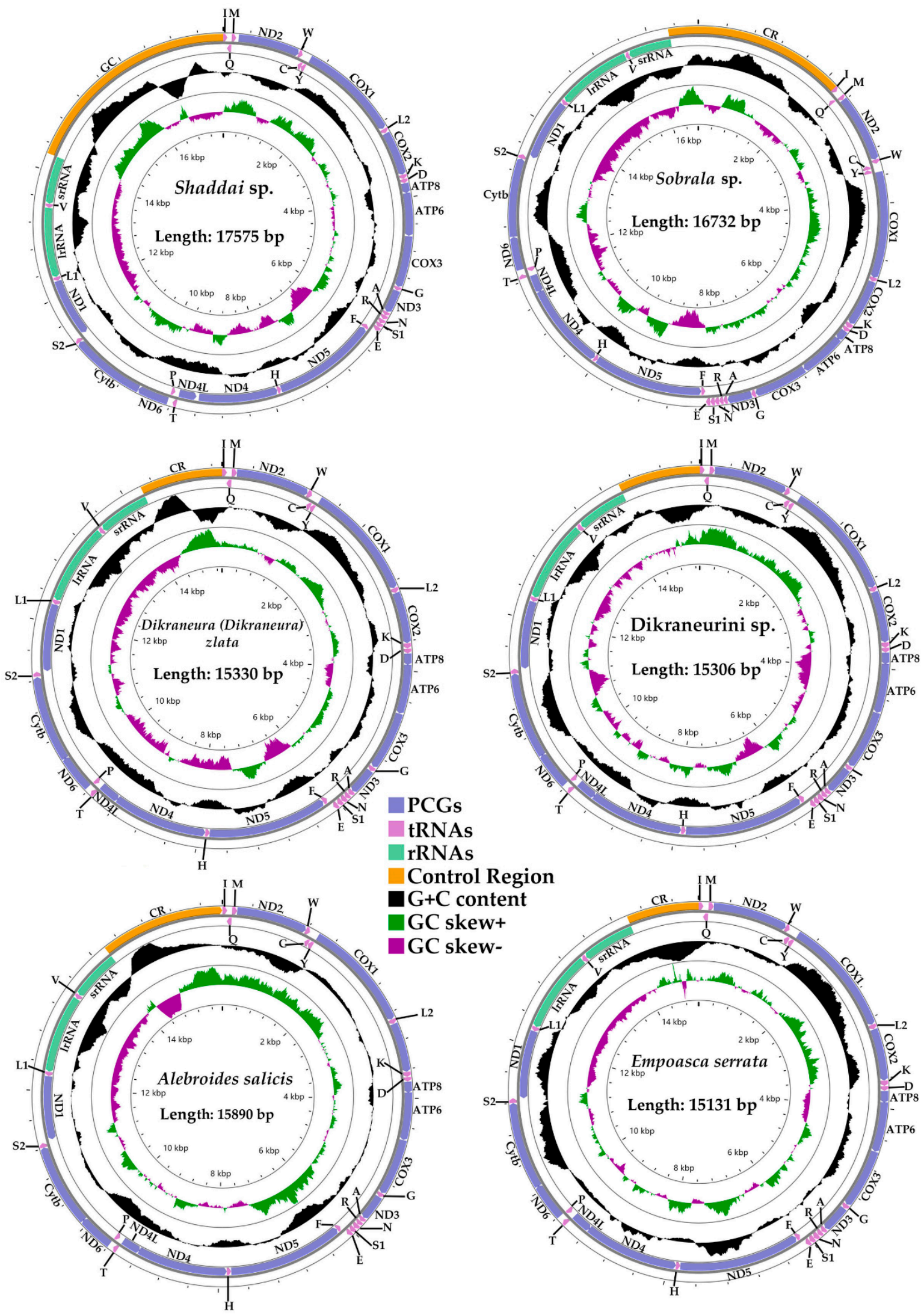

Figure 1. Circular map of the mitochondrial genome of Shaddai sp., Sobrala sp., Dikraneura (Dikraneura) zlata, Dikraneurini sp., Alebroides salici and Empoasca serrata. 


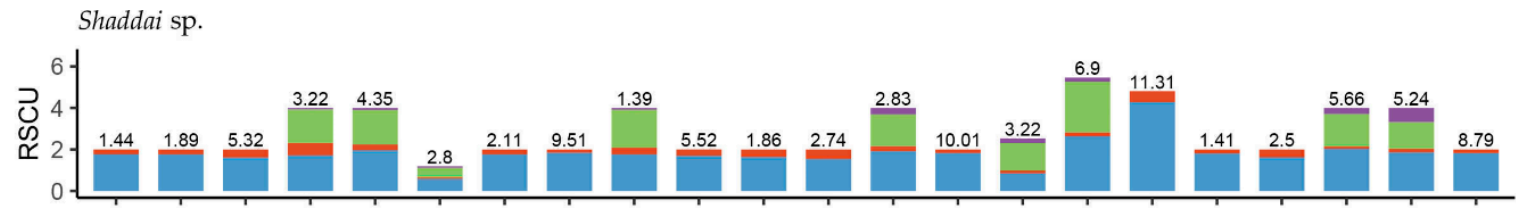

Sobrala sp.

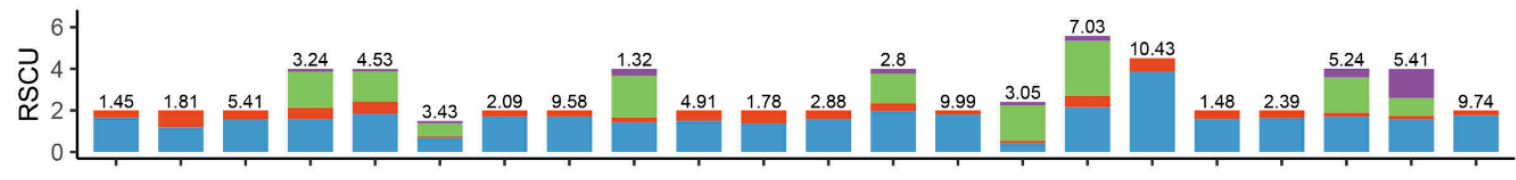

Ghauriana sinensis
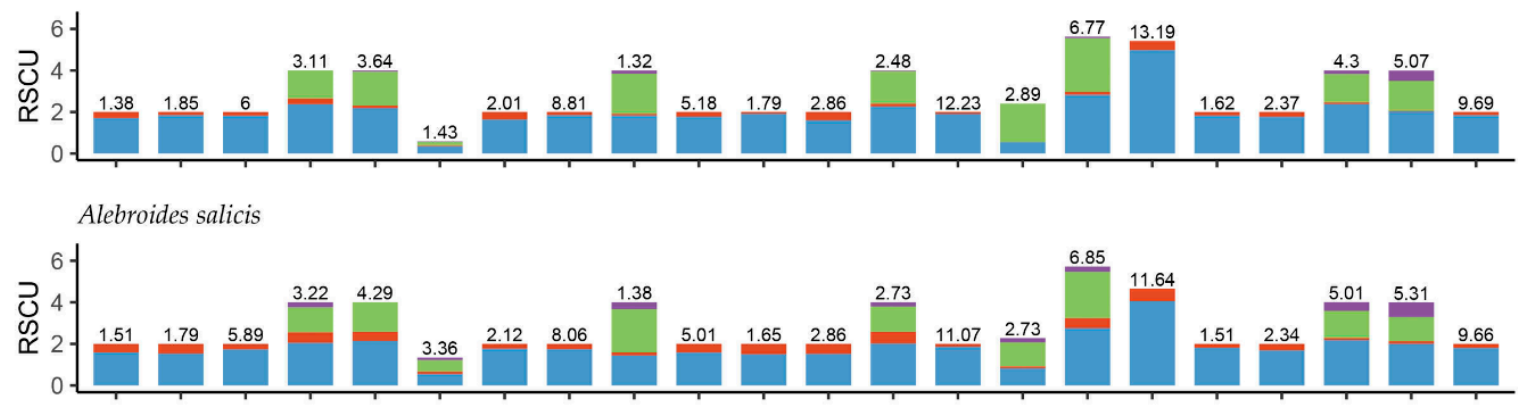

Empoasca serrata
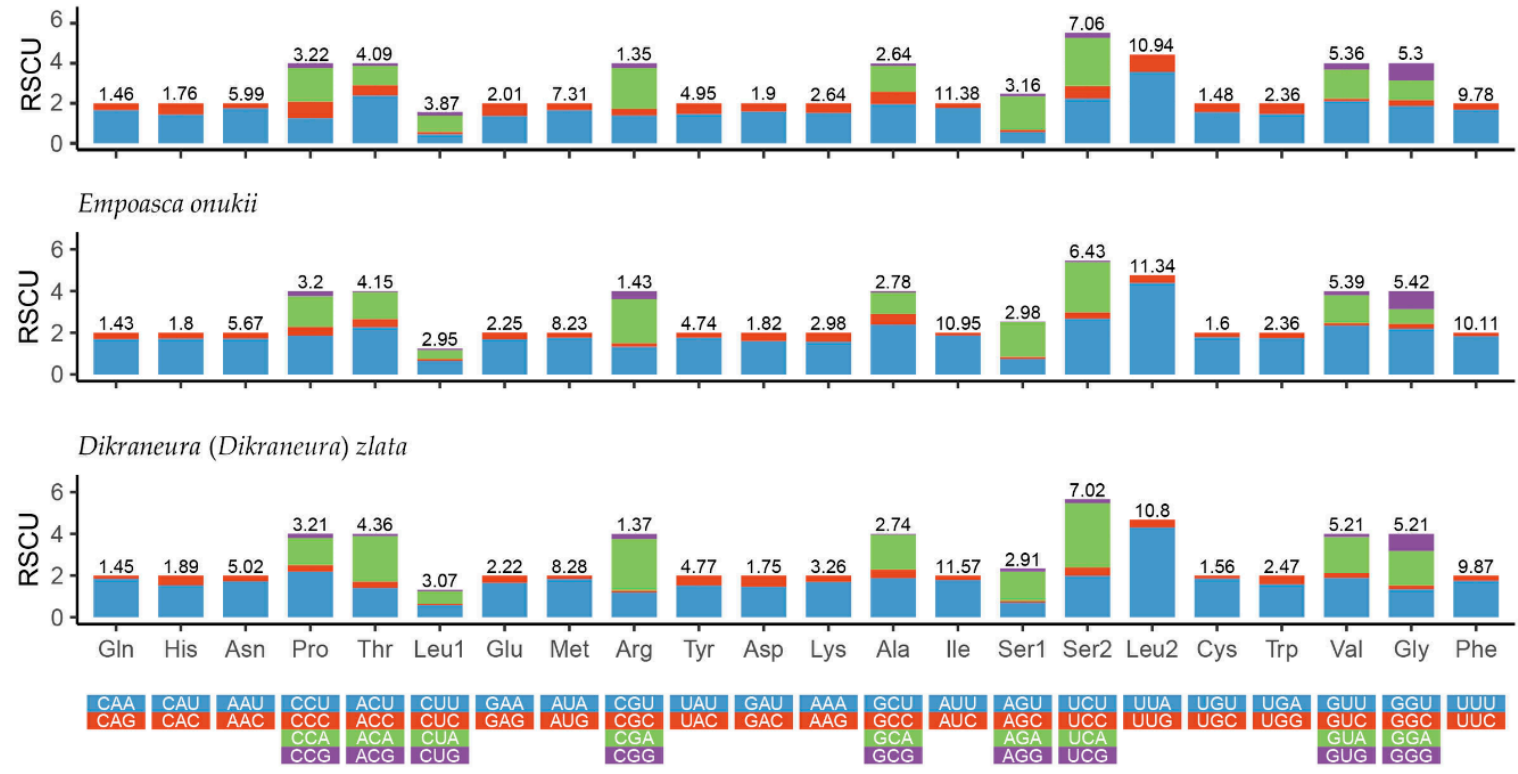

Figure 2. Relative synonymous codon usage (RSCU) in the mitogenomes of Shaddai sp., Sobrala sp., Alebroides salicis, Empoasca serrata, Empoasca onukii and Dikraneura (D.) zlata.

\subsection{Transfer and Ribosomal RNA Genes}

Each of the complete mitogenomes is composed of 22 tRNA genes and 2 rRNAs. The positions of all 22 tRNAs are dispersed throughout the whole mitogenomes, and the two rRNA were identified by the boundary of the adjacent genes and based on the comparison with homologous genes from other Typhlocybinae species. The total length of tRNAs among the 11 newly sequenced mitogenomes ranges from 1383 to $1455 \mathrm{bp}$, while the total length of rRNAs ranges from 1805 to $1956 \mathrm{bp}$. Both kinds of total RNAs present a negative AT-skew and positive GC-skew. Meanwhile, with a heavy AT nucleotide bias, the AT content attains 76.1-79.0\% in total tRNAs, while in total rRNAs the AT content reaches $79.8-83.0 \%$, which is higher than that in total tRNAs (Table S4). As presented in Figures 3 and S4-S13, all tRNAs folded into the typical clover-leaf secondary structure, 
except for some tRNAs with a reduced arm, presenting a simple loop or composed of unpaired bases. Examples include the DHU arm of trnS1 in Shaddai sp., Sobrala sp., Dikraneurini sp., Yangisunda tiani and TYC arm of trnQ in Sobrala sp. presenting a simple loop, commonly present in the previously sequenced mitogenomes of Cicadellidae. However, $\operatorname{trnR}$ is the most inconsistent among all the tRNAs due to the acceptor arm being composed of unpaired bases, such as the acceptor arm of trnR in Elbelus tripunctatus and Yangisunda tiani only having 4 or $5 \mathrm{bp}$, Dikraneura (D.) zlata and Eurhadina jarrary acceptor arm of $\operatorname{trnR}$ having 3 or $6 \mathrm{bp}$, Shaddai sp. where the acceptor arm of $\operatorname{trnR}$ has 6 or $7 \mathrm{bp}$. These unusual features are seldomly reported in Typhlocybinae [22]. Unpaired bases are also found in the TYC arm of trnQ in Shaddai sp. and the anticodon arm of trnS1 in Sobrala sp. and Dikraneurini sp. All of the above unusual characteristics may reveal mitogenome divergences among different species [49].

From Figures 3 and S4-S13, we observed that all tRNAs of the 11 newly sequenced mitogenomes are highly conserved in lengths of $7 \mathrm{bp}$ for the acceptor arm, $7 \mathrm{bp}$ for the anticodon loop, and $5 \mathrm{bp}$ for the anticodon arm, while the lengths of the variable loop, DHU and TYC arms are fickle. Additionally, we recognize a total of eight types of unmatched base pairs in the arms of tRNAs from the figures $(A-C, A-G, U-C, U-G, A-A, U-U, C-C$, $\mathrm{G}-\mathrm{G})$, of which $\mathrm{C}-\mathrm{C}$ and $\mathrm{G}-\mathrm{G}$ unmatched base pairs have seldomly been reported in previous mitogenomes of Typhlocybinae. The total number of unmatched base pairs found was 47 in Shaddai sp., 34 in Sobrala sp., 30 in Alebroides salicis, 35 in Empoasca serrata, 40 in Dikraneurini sp., 35 in Dikraneura (D.) zlata, 47 in Kaukania anser, 57 in Elbelus tripunctatus, 48 in Eupteryx (E.) adspersa, 47 in Eurhadina jarrary and 42 in Yangisunda tiani.

\subsection{Control Region}

The control region, also named the A + T-rich region, is supposed to participate in regulation of transcription and replication [10-12]. Located at the position between $r r n S$ and $t r n I$, it is the longest intergenic spacer in the mitogenome, with AT content ranging from $59.3 \%$ (Alebroides salicis) to $93.8 \%$ (Yangisunda tiani) and length ranging from 920 (Yangisunda tiani) to 3328 bp (Shaddai sp.) among the 11 newly sequenced mitogenomes. The nucleotide composition of the control region presents a positive AT skew and negative GC skew in Shaddai sp., Sobrala sp., Dikraneura (D.) zlata, Dikraneurini sp., Alebroides salicis, Elbelus tripunctatus, Eurhadina jarrary and Yangisunda tiani, while the rest of the species present a contrary strand asymmetry (Table S4). All of the control regions of the 11 newly sequenced mitogenomes have tandem repeats, except for Elbelus tripunctatus. The fragment lengths, nucleotide sequences, positions and copy numbers of repeat units are highly variable (Figure 4).

\subsection{Gene Overlaps and Intergenic Spacers}

Table S6 reveals that for the numbers ranging from 10 to 16 in each of the $11 \mathrm{mi}-$ togenomes, the longest overlap is 10 bp between trnS2 and nad1 in Dikraneurini sp., and the gene overlaps appear more frequently between tRNAs, which is of interest concerning the lesser evolutionary restraints of tRNAs [50].

We recognize 6-14 intergenic spacers in each of the 11 mitogenomes, with lengths ranging from 1 to $57 \mathrm{bp}$. The longest intergenic spacer was found in Elbelus tripunctatus, between nad5 and trnH. The intergenic spacers can be found in most mitogenomes, presumed to be essential for the transcriptional machinery to recognize the transcription termination site [51,52]. 

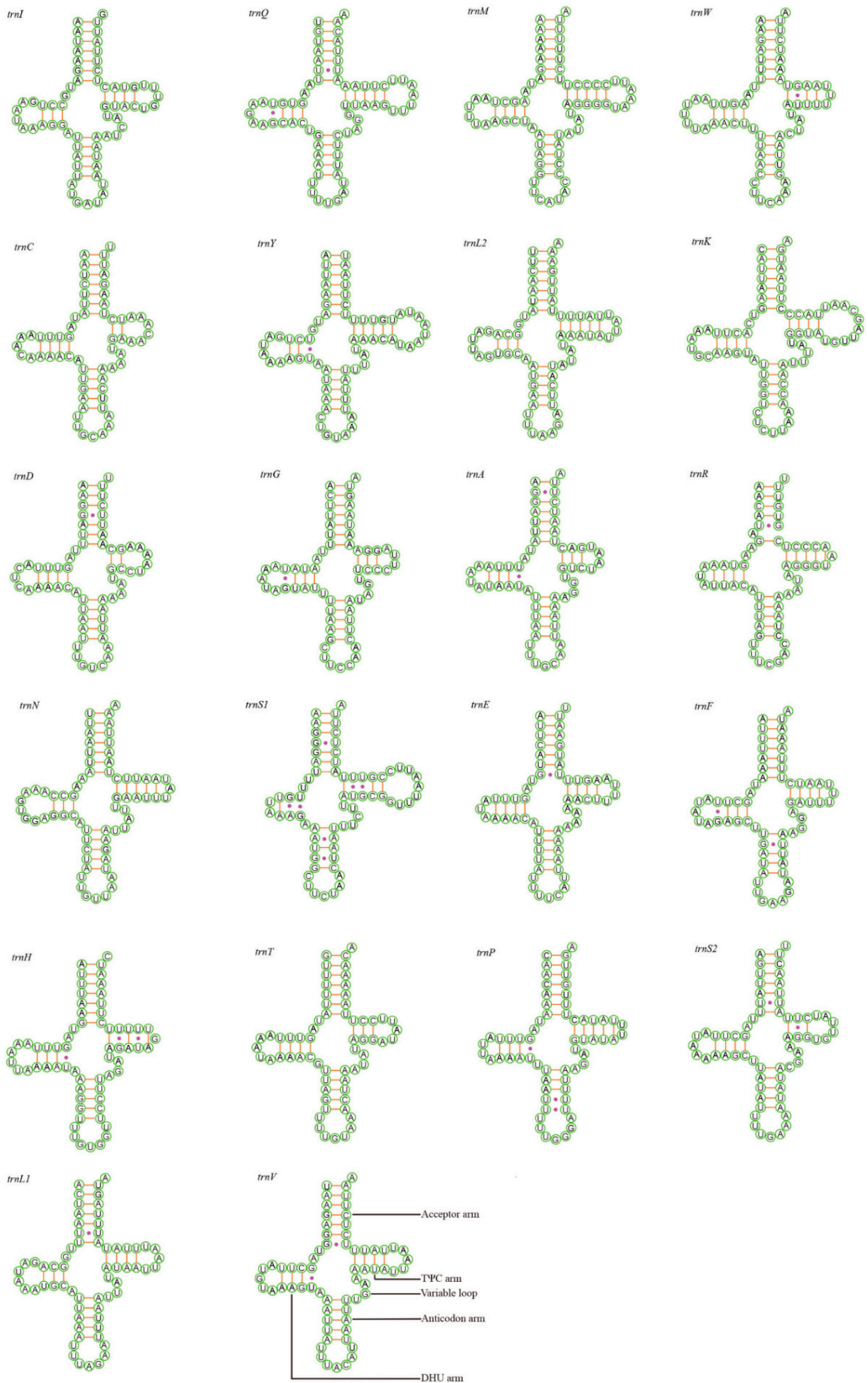

Figure 3. Predicted secondary cloverleaf structure for the tRNAs of Alebroides salicis. 
Shaddai sp.

$3328 \mathrm{bp}$

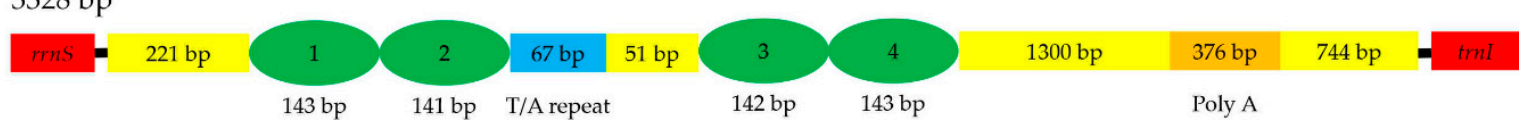

Sobrala sp.

$2601 \mathrm{bp}$

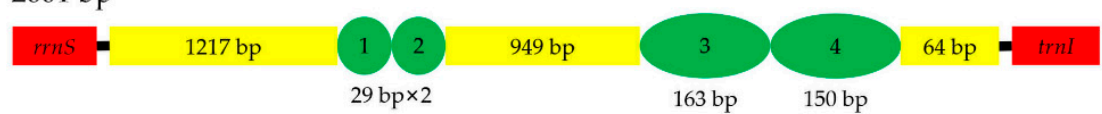

Dikraneura (Dikraneura) zlata

$1085 \mathrm{bp}$

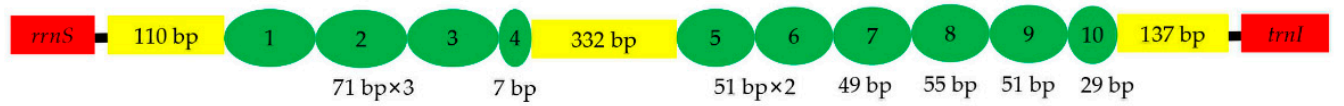

Dikraneurini sp.

1080bp
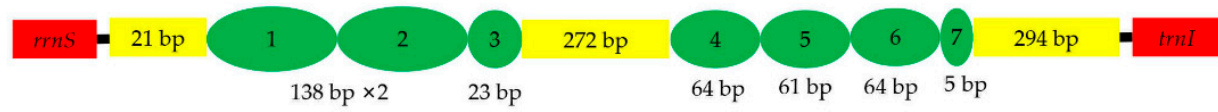

Alebroides salicis

$1697 \mathrm{bp}$

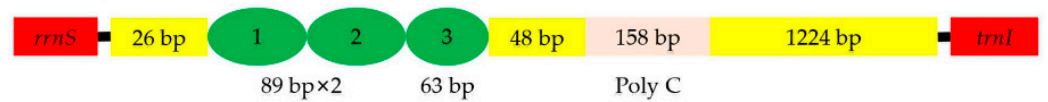

Empoasca serrata

$935 \mathrm{bp}$

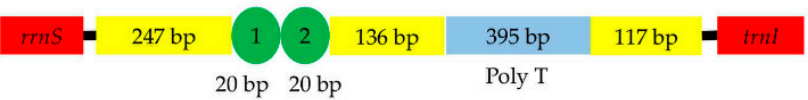

Kaukania anser

$982 \mathrm{bp}$

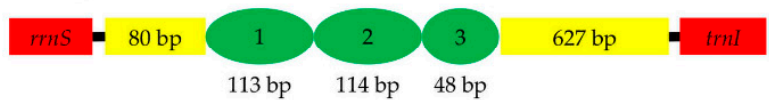

Eupteryx (Eupteryx) adspersa

$923 \mathrm{bp}$

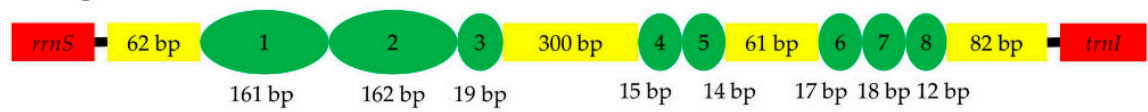

Eurhadina jarrary

989 bp

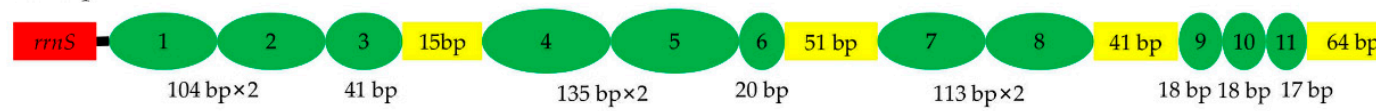

Yangisunda tiani

920bp

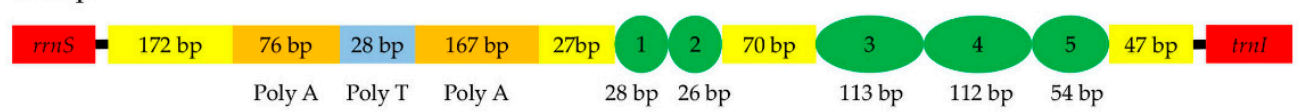

Figure 4. Organization of the control regions in the mitochondrial genomes of Shaddai sp., Sobrala sp., Dikraneura (Dikraneura) zlata, Dikraneurini sp., Alebroides salicis, Empoasca serrata, Kaukania anser, Eupteryx (Eupteryx) adspersa, Eurhadina jarrary and Yangisunda tiani. The green ovals indicate the tandem repeats; the yellow boxes indicate the non-repeat regions. 


\subsection{Nucleotide Diversity and Evolutionary Rate Analysis}

The nucleotide diversity (Pi values) based on 13 PCGs among the 22 Typhlocybinae mitogenomes was computed by the sliding window analysis. The result is exhibited in Figure 5A, showing that the Pi values of 13 PCGs range from 0.206 to 0.382 , of which, atp 8 and nad 2 have relatively high Pi values of 0.382 and 0.374 indicating the highest variability. The genes cox 1 and nad1 have relatively low Pi values of 0.206 and 0.235 , indicating that they are the most conserved genes among the 13 PCGs. Pairwise genetic distance analysis was also conducted based on 13 PCGs among the 22 Typhlocybinae mitogenomes. The congruent results (Figure $5 \mathrm{~B}$ ) show genetic distances ranging from 0.24 to 0.53 , of which, atp 8 and nad 2 have relatively high distances of both 0.53 , indicating the fastest evolution, while cox 1 and nad1 have relatively low distances of 0.24 and 0.28 , indicating the slowest evolution. Average non-synonymous (Ka) and synonymous (Ks) substitution rates $(\mathrm{Ka} / \mathrm{Ks})$ analyses were used to estimate the evolutionary rate of each of the 13 PCGs among the 22 Typhlocybinae mitogenomes. Ka/Ks rates ranged from 0.16 to 0.84 , between 0 and 1, meaning that all 13 PCGs are evolving under the purifying selection. Among the 13 PCGs, atp8 and nad2 have relatively high $\mathrm{Ka} / \mathrm{Ks}$ rates of 0.84 and 0.62 indicates the weakest purifying pressure, while cox 1 and $c y t b$ have relatively low $\mathrm{Ka} / \mathrm{Ks}$ rates of 0.16 and 0.24 indicates the strongest purifying pressure.

The cox1 gene is one of the most universal markers for identifying species and analyzing the phylogenetic relationships of leafhoppers [53-55], but it has the lowest Pi values, genetic distance and $\mathrm{Ka} / \mathrm{Ks}$ rates, indicating it is the most conserved gene within the 13 PCGs among the 22 Typhlocybinae mitogenomes. Meanwhile, atp 8 and nad 2 are the fastest evolving genes, suggesting that atp 8 and nad 2 would be two ideal candidate markers for sibling species delimitation and population genetic differentiation in Typhlocybinae.

\subsection{Phylogenetic Relationships}

The phylogenetic relationships of 22 Typhlocybinae species and two outgroup species were reconstructed by the ML and BI methods under the best models, based on three datasets, PCG123, PCG123R and PCG12, and are almost congruent. Furthermore, most nodes indicate strong support (Figures 6, S14 and S15). The 22 typhlocybine species used in this study formed a monophyletic group with respect to the two outgroup species. Additionally, Alebrini, Empoascini, Dikraneurini and Erythroneurini are also recovered as monophyletic clades, respectively, and relationships among the tribes are well resolved. However, all members of Typhlocybini and Zyginellini are clustered into a clade, indicating that the two groups are mutually paraphyletic. This is similar to the studies of Ahmed (1983), Dietrich (2013) and Chen (2021) to some extent, that divided Typhlocybinae into five tribes: Alebrini, Dikraneurini (=Forcipatini), Empoascini (=Jorumini), Erythroneurini and Typhlocybini (=Eupterygini, Zyginellini) but different from the studies of Dworakowska (1979) and Zhang (1990) based on morphological data that divided Typhlocybinae into six tribes (Alebrini, Dikraneurini, Empoascini, Erythroneurini, Typhlocybini and Zyginellini) $[3,8,9,56,57]$, although this is currently the most widely held tribal classification system of Typhlocybinae. Some other tribal classification of Typhlocybinae having recognized 4-10 tribes that are too different from ours to be acceptable [58,59]. Here in our study, we support Zyginellini as a synonym of Typhlocybini, as treated or as the result revealed in phylogenetic studies based on molecular data of Typhlocybinae $[4,8,22,57,60]$. 

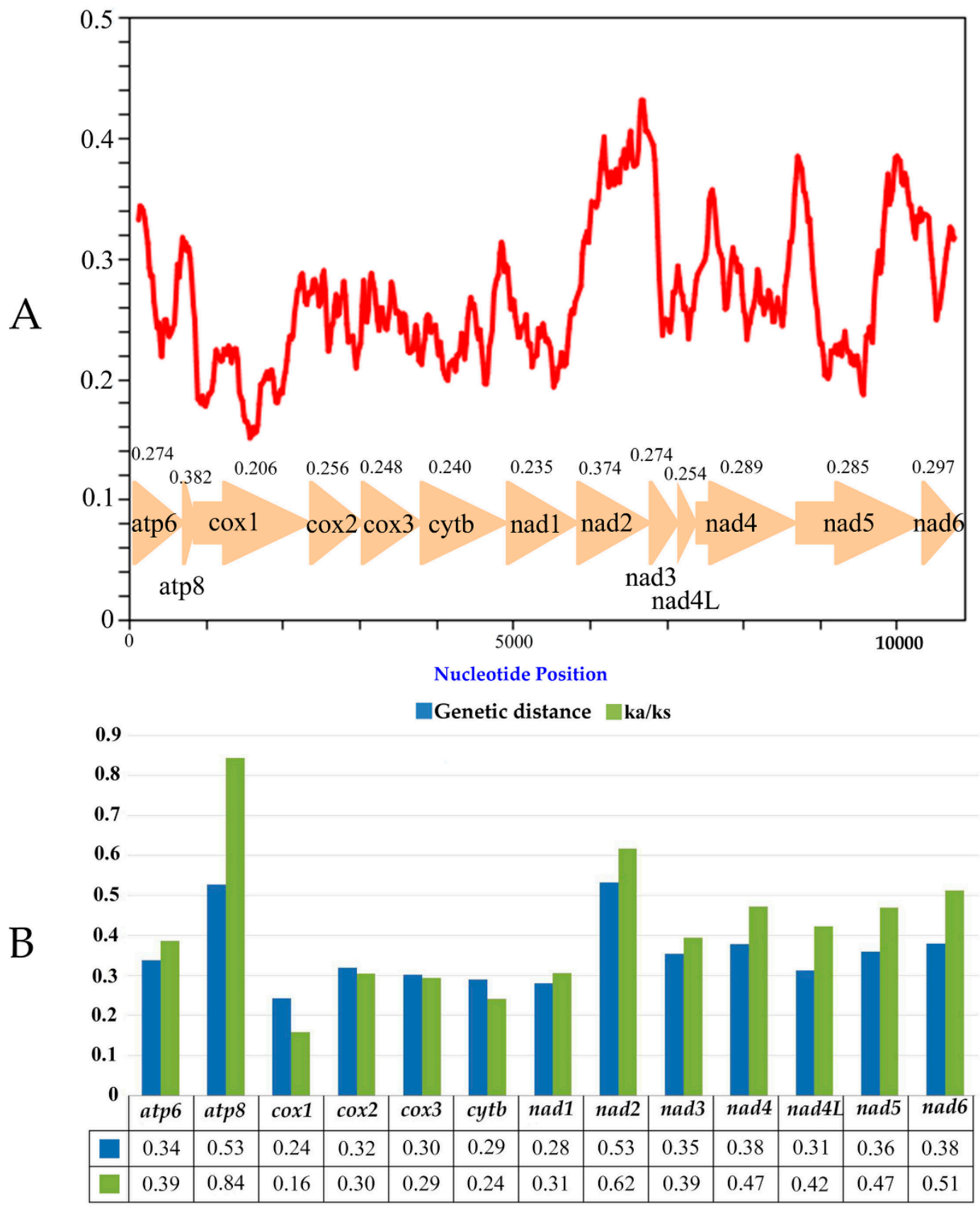

Figure 5. (A) Sliding window analyses of 13 PCGs among 22 species of Typhlocybinae, the red line shows the value of nucleotide diversity Pi. (B) Genetic distances and the ratio of non-synonymous (Ka) to synonymous (Ks) substitution rates of 13 PCGs among 22 species of Typhlocybinae, the average value for each PCG is shown under the gene name. 


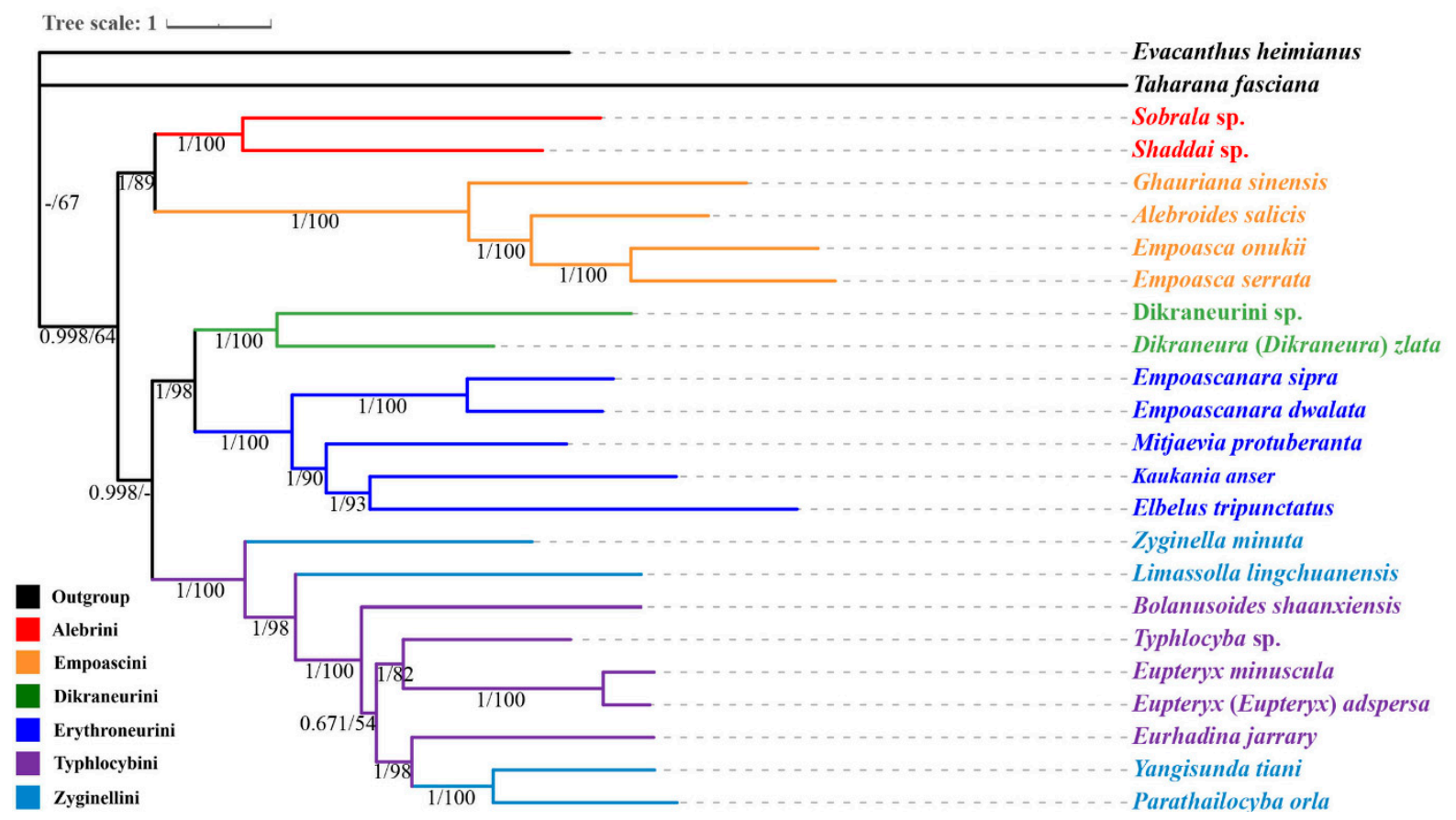

Figure 6. The phylogenetic tree produced using BI methods based on the dataset of PCG123. ML and BI analyses showed the same topology. The numbers under the branches are Bayesian posterior probabilities (PP) and bootstrap support values (BS).

Inter-tribe relationships of the five monophyletic tribes are identical to the one obtained in the Anchored Hybrid Enrichment (AHE) based phylogenomics [6], with Alebrini sister to Empoascini as the basal branch, followed by the branch of Dikraneurini sister to Erythroneurini, and finally the branch with members of Typhlocybini and Zyginellini, indicating the relationships among the five monophyletic tribes are to some extent stable. It is different from the sister-group relationship of Empoascini to Dikraneurini that proposed by Zhang (1990) and Xu (2021) based on morphological data and Dikraneurini to Typhlocybini and Zyginellini proposed by Chen (2021) based on molecular data [9,57,61]. Therefore, Typhlocybinae divides into four tribes (Alebrini, Dikraneurini, Empoascini and Typhlocybini) and Erythroneurini as a subtribe of Dikraneurini as proposed by Balme based on morphological data and 16S rRNA with histone [4] are supported. To sum up, distinguishing relationships based solely upon morphological data is not reliable. Nevertheless, we did not verify that Empoascini was not to be as uniform as previously believed and Typhlocybini can be subdivided into subtribes $[60,62]$, a broader analysis with more mitogenomes of representative samples is necessary to confirm these results and to elucidate the status of Typhlocybinae within Membracoidea.

\section{Conclusions}

In our study, 11 complete mitogenomes were newly sequenced and the phylogenetic relationships of the six tribes (Alebrini, Dikraneurini, Empoascini, Erythroneurini, Typhlocybini and Zyginellini) within Typhlocybinae were comparatively analyzed. Compared to other previously reported complete mitogenomes, all of these complete mitogenomes with number and order of the genes were highly conserved in overall organization. The PCGs initiate with ATN/TTG/GTG, and terminate with TAA/TAG/T. All tRNAs fold into the typical clover-leaf secondary structure, except for some tRNAs with a reduced arm, presenting a simple loop or composed of unpaired bases. Ka/Ks and genetic distance analyses indicate that the atp 8 and nad2 exhibit the highest evolutionary rate among all of the PCGs. Phylogenetic analyses based on whole mitogenome sequences, with three different datasets (PCG123, PCG123R, PCG12), using both maximum likelihood and Bayesian methods, indicated the monophyly of Typhlocybinae and its inner tribes, respectively, 
except for Typhlocybini and Zyginellini being mutually paraphyletic. Topologies of the monophyletic tribes/subtribes showed Alebrini sister to Empoascini as the basal branch, followed by branch of Dikraneurini and Erythroneurini, and finally Typhlocybini. Our study provided the valuable data and efficient framework for the future phylogenetic research of Typhlocybinae.

Supplementary Materials: The following are available online at https:/ /www.mdpi.com/article/10 .3390/insects12080678/s1. Table S1. Collection information of adult specimens in this study; Table S2. The best partitioning schemes and models for Maximum likelihood (ML) method based on four datasets selected by PartitionFinder; Table S3. The best partitioning schemes and models for Bayesian inference (BI) method based on four datasets selected by PartitionFinder; Table S4. Nucleotide composition and skewness of eleven Typhlocybinae spp. mitogenomes; Table S5. Start and stop codons of the mitochondrial genomes; Table S6. Overlapping Sequences and Intergenic Spacers. Figure S1. Circular map of the mitochondrial genome of Elbelus tripunctatus, Kaukania anser, Eupteryx (Eupteryx) adspersa, Eurhadina jarrary and Yangisunda tiani; Figure S2. Relative synonymous codon usage (RSCU) in the mitogenomes of Dikraneurini sp., Empoascanara dwalata, Empoascanara sipra, Mitjaevia protuberanta, Elbelus tripunctatus, Kaukania anser, Zyginella minuta, Limassolla lingchuanensis and Bolanusoides shaanxiensis. Figure S3. Relative synonymous codon usage (RSCU) in the mitogenomes of Typhlocyba sp., Eupteryx (E.) adspersa, Eupteryx minuscula, Eurhadina jarrary, Parathailocyba orla and Yangisunda tiani. Figure S4. Predicted secondary cloverleaf structure for the tRNAs of Dikraneura (D.) zlata. Figure S5. Predicted secondary cloverleaf structure for the tRNAs of Elbelus tripunctatus. Figure S6. Predicted secondary cloverleaf structure for the tRNAs of Empoasca serrata. Figure S7. Predicted secondary cloverleaf structure for the tRNAs of Eupteryx (E.) adspersa. Figure S8. Predicted secondary cloverleaf structure for the tRNAs of Eurhadina jarrary. Figure S9. Predicted secondary cloverleaf structure for the tRNAs of Kaukania anser. Figure S10. Predicted secondary cloverleaf structure for the tRNAs of Dikraneurini sp. Figure S11. Predicted secondary cloverleaf structure for the tRNAs of Shaddai sp. Figure S12. Predicted secondary cloverleaf structure for the tRNAs of Sobrala sp. Figure S13. Predicted secondary cloverleaf structure for the tRNAs of Yangisunda tiani.

Author Contributions: Conceptualization, S.L., M.H. and Y.Z.; methodology, S.L.; formal analysis, S.L. and M.H.; writing—original draft preparation, S.L. and Y.Z.; writing—review and editing, S.L., Y.Z., and M.H.; supervision, Y.Z.; project administration, Y.Z. and M.H.; funding acquisition, Y.Z. and M.H. All authors have read and agreed to the published version of the manuscript.

Funding: This study was supported by the National Natural Science Foundation of China (31420103911, 32070478, 31372233) and The Ministry of Science and Technology of the People's Republic of China (2015FY210300, 2005DKA21402).

Institutional Review Board Statement: Not applicable.

Informed Consent Statement: Not applicable.

Data Availability Statement: Data available on request.

Acknowledgments: We extend our heartfelt gratitude to J. R. Schrock, Emporia State University, Kansas, USA, for reviewing the manuscript. Sincere thanks to Deliang $\mathrm{Xu}$ for his assistance in some of the programs used for the analyses and Meixia Yang for her great help in obtaining specimens. We are also grateful to Luyao Ma, Weijian Huang, Jiu Tang, Xian Zhou and Wenqian Wang for their kind advice in analyses.

Conflicts of Interest: The authors declare no conflict of interest.

\section{References}

1. Dietrich, C.H.; Dmitriev, D.A. Review of the New World genera of the leafhopper tribe Erythroneurini (Hemiptera: Cicadellidae: Typhlocybinae). Bull. Ill. Nat. Hist. Surv. 2006, 37, 119-190. [CrossRef]

2. Dietrich, C.H.; Dmitriev, D.A. Review of the New World Erythroneurini (Hemiptera: Cicadellidae: Typhlocybinae). II. Genus Zyginama. Bull. Ill. Nat. Hist. Surv. 2008, 38, 129-175. [CrossRef]

3. Dworakowska, I. The leafhopper tribe Zyginellini (Homoptera, Auchenorrhyncha, Cicadellidae, Typhlocybinae). Rev. Zool. Afr. 1979, 93, 299-331.

4. Balme, G.R. In Phylogeny and Systematics of the Leafhopper Subfamily Typhlocybinae (Insecta: Hemiptera: Typhlocybinae). Ph.D. Thesis, North Carolina State University, Raleigh, NC, USA, 2007. 
5. Dietrich, C.H. Phylogeny of the leafhopper subfamily Evacanthinae with a review of Neotropical species and notes on related groups (Hemiptera: Membracoidea: Cicadellidae). Syst. Entomol. 2004, 29, 455-487. [CrossRef]

6. $\quad$ Dietrich, C.H.; Allen, J.M.; Lemmon, A.R.; Lemmon, E.M.; Takiya, D.M.; Evangelista, O.; Walden, K.K.; Grady, P.G.; Johnson, K.P.; Wiegmann, B. Anchored hybrid enrichment-based phylogenomics of leafhoppers and treehoppers (Hemiptera: Cicadomorpha: Membracoidea). Insect Syst. Divers. 2017, 1, 57-72. [CrossRef]

7. Dietrich, C.H.; Rakitov, R.A.; Holmes, J.L.; Black, W.C. Phylogeny of the major lineages of Membracoidea (Insecta: Hemiptera: Cicadomorpha) based on $28 \mathrm{~S}$ rDNA Sequences. Mol. Phylogenet. Evol. 2001, 18, 293-305. [CrossRef] [PubMed]

8. Dietrich, C.H. South American leafhoppers of the tribe Typhlocybini (Hemiptera: Cicadellidae: Typhlocybinae). Zoologia 2013, 30, 519-568. [CrossRef]

9. Zhang, Y.L. A Taxonomic Study of Chinese Cicadellidae (Homoptera); Tianze Eldonejo: Yangling, China, 1990.

10. Boore, J.L. Animal mitochondrial genomes. Nucleic Acids Res. 1999, 27, 1767-1780. [CrossRef]

11. Cameron, S.L. Insect mitochondrial genomics: Implications for evolution and phylogeny. Annu. Rev. Entomol. 2014, 59, 95-117. [CrossRef]

12. Zhang, D.X.; Hewitt, G.M. Insect mitochondrial control region: A review of its structure, evolution and usefulness in evolutionary studies. Biochem. Syst. Ecol. 1997, 25, 99-120. [CrossRef]

13. Wolstenholme, D.R. Genetic novelties in mitochondrial genomes of multicellular animals. Curr. Opin. Genet. Dev. 1992, 2, 918-925. [CrossRef]

14. Wolstenholme, D.R. Animal mitochondrial DNA: Structure and evolution. Int. Rev. Cytol. Surv. Cell Biol. 1992, $141,173-216$.

15. Du, Z.; Hasegawa, H.; Cooley, J.R.; Simon, C.; Yoshimura, J.; Cai, W.; Sota, T.; Li, H. Mitochondrial genomics reveals shared phylogeographic patterns and demographic history among three periodical cicada species groups. Mol. Biol. Evol. 2019, 36, 1187-1200. [CrossRef] [PubMed]

16. Huang, W.; Zhang, Y. Characterization of two complete mitochondrial genomes of Ledrinae (Hemiptera: Cicadellidae) and phylogenetic analysis. Insects 2020, 11, 609. [CrossRef] [PubMed]

17. Li, H.; Leavengood, J.M., Jr.; Chapman, E.G.; Burkhardt, D.; Song, F.; Jiang, P.; Liu, J.; Zhou, X.; Cai, W. Mitochondrial phylogenomics of Hemiptera reveals adaptive innovations driving the diversification of true bugs. Proc. R. Soc. B Biol. Sci. 2017, 284, 20171223. [CrossRef] [PubMed]

18. Lv, L.; Peng, X.X.; Jing, S.L.; Liu, B.F.; Zhu, L.L.; He, G.C. Intraspecific and interspecific variations in the mitochondrial genomes of Nilaparvata (Hemiptera: Delphacidae). J. Econ. Entomol. 2015, 108, 2021-2029. [CrossRef]

19. Song, N.; Liang, A.P.; Bu, C.P. A molecular phylogeny of Hemiptera inferred from mitochondrial genome sequences. PLoS ONE 2012, 7, e48778. [CrossRef]

20. Song, N.; Liang, A. The complete mitochondrial genome sequence of Geisha distinctissima (Hemiptera: Flatidae) and comparison with other hemipteran insects. Acta Biochim. Biophys. Sin. 2009, 41, 206-216. [CrossRef]

21. Xu, D.; Yu, T.; Zhang, Y. Characterization of the complete mitochondrial genome of Drabescus inefffectus and Roxasellana stellata (Hemiptera: Cicadellidae: Deltocephalinae: Drabescini) and Their Phylogenetic Implications. Insects 2020, 11, 534. [CrossRef]

22. Zhou, X.; Dietrich, C.H.; Huang, M. Characterization of the complete mitochondrial genomes of two species with preliminary investigation on phylogenetic status of Zyginellini (Hemiptera: Cicadellidae: Typhlocybinae). Insects 2020, 11, 684. [CrossRef]

23. Kearse, M.; Moir, R.; Wilson, A.; Stones-Havas, S.; Cheung, M.; Sturrock, S.; Buxton, S.; Cooper, A.; Markowitz, S.; Duran, C. Geneious basic: An integrated and extendable desktop software platform for the organization and analysis of sequence data. Bioinformatics 2012, 28, 1647-1649. [CrossRef]

24. Bernt, M.; Donath, A.; Jühling, F.; Externbrink, F.; Florentz, C.; Fritzsch, G.; Pütz, J.; Middendorf, M.; Stadler, P.F. MITOS: Improved de novo metazoan mitochondrial genome annotation. Mol. Phylogenet. Evol. 2013, 69, 313-319. [CrossRef]

25. Benson, G. Tandem repeats finder: A program to analyze DNA sequences. Nucleic Acids Res. 1999, 27, 573-580. [CrossRef] [PubMed]

26. Grant, J.R.; Stothard, P. The CGView Server: A comparative genomics tool for circular genomes. Nucleic Acids Res. 2008, 36, W181-W184. [CrossRef]

27. Zhang, D.; Gao, F.L.; Jakovli 'c, I.; Zou, H.; Zhang, J.; Li, W.X.; Wang, G.T. PhyloSuite: An integrated and scalable desktop platform for streamlined molecular sequence data management and evolutionary phylogenetics studies. Mol. Ecol. Resour. 2020, 20, 348-355. [CrossRef] [PubMed]

28. Perna, N.T.; Kocher, T.D. Patterns of nucleotide composition at fourfold degenerate sites of animal mitochondrial genomes. J. Mol. Evol. 1995, 41, 353-358. [CrossRef]

29. Rozas, J.; Ferrer-Mata, A.; Sanchez-DelBarrio, J.C.; Guirao-Rico, S.; Librado, P.; Ramos-Onsins, S.E.; Sanchez-Gracia, A. DnaSP 6: DNA sequence polymorphism analysis of large data sets. Mol. Biol. Evol. 2017, 34, 3299-3302. [CrossRef] [PubMed]

30. Kumar, S.; Stecher, G.; Li, M.; Knyaz, C.; Tamura, K. MEGA X: Molecular Evolutionary Genetics Analysis across computing platforms. Mol. Biol. Evol. 2018, 35, 1547-1549. [CrossRef] [PubMed]

31. Wang, J.J.; Yang, M.F.; Dai, R.H.; Li, H. Complete mitochondrial genome of Evacanthus heimianus (Hemiptera: Cicadellidae: Evacanthinae) from China. Mitochondrial DNA Part B 2019, 4, 284-285. [CrossRef]

32. Wang, J.J.; Dai, R.H. Complete mitochondrial genome of Taharana fasciana (Insecta, Hemiptera: Cicadellidae) and comparison with other Cicadellidae insects. Genetica 2017, 145, 593-602. [CrossRef] 
33. Shi, R.; Yu, X.F.; Yang, M.F. Complete mitochondrial genome of Ghauriana sinensis (Hemiptera: Cicadellidae: Typhlocybinae). Mitochondrial DNA Part B 2020, 5, 1367-1368. [CrossRef]

34. Liu, J.H.; Sun, C.Y.; Long, J.; Guo, J.J. Complete mitogenome of tea green leafhopper, Empoasca onukii (Hemiptera: Cicadellidae) from Anshun, Guizhou Province in China. Mitochondrial DNA Part B 2017, 2, 808-809. [CrossRef] [PubMed]

35. Chen, X.X.; Yuan, Z.W.; Li, C.; Song, Y.H. Complete mitochondrial genome sequence of Empoascanara dwalata (Hemiptera: Cicadellidae: Typhlocybinae). Mitochondrial DNA Part B 2020, 5, 2260-2261. [CrossRef] [PubMed]

36. Tan, C.; Chen, X.X.; Li, C.; Song, Y.H. The complete mitochondrial genome of Empoascanara sipra (Hemiptera: Cicadellidae: Typhlocybinae) with phylogenetic consideration. Mitochondrial DNA Part B 2020, 5, 260-261. [CrossRef] [PubMed]

37. Yuan, X.; Li, C.; Song, Y.H. Characterization of the complete mitochondrial genome of Mitjaevia protuberanta (Hemiptera: Cicadellidae: Typhlocybinae). Mitochondrial DNA Part B 2020, 5, 601-602. [CrossRef]

38. Han, C.; Yan, B.; Yu, X.F.; Yang, M.F. Complete mitochondrial genome of Zyginella minuta (Cicadellidae: Typhlocybinae: Zyginellini) from China, with its phylogenetic analysis. Mitochondrial DNA Part B 2020, 5, 2795-2796. [CrossRef]

39. Yuan, X.W.; Xiong, K.N.; Li, C.; Song, Y.H. The complete mitochondrial genome of Limassolla lingchuanensis (Hemiptera: Cicadellidae: Typhlocybinae). Mitochondrial DNA Part B 2020, 5, 229-230. [CrossRef]

40. Jiang, J.; Yuan, X.; Yuan, Z.; Song, Y.H. The complete mitochondrial genome of Parathailocyba orla (Hemiptera: Cicadellidae: Typhlocybinae). Mitochondrial DNA Part B 2020, 5, 1981-1982. [CrossRef]

41. Song, N.; Cai, W.Z.; Li, H. Deep-level phylogeny of Cicadomorpha inferred from mitochondrial genomes sequenced by NGS. Sci. Rep. 2017, 7, 10429. [CrossRef] [PubMed]

42. Katoh, K.; Standley, D.M. MAFFT multiple sequence alignment software version 7: Improvements in performance and usability. Mol. Biol. Evol. 2013, 30, 772-780. [CrossRef] [PubMed]

43. Talavera, G.; Castresana, J. Improvement of phylogenies after removing divergent and ambiguously aligned blocks from protein sequence alignments. Syst. Biol. 2007, 56, 564-577. [CrossRef]

44. Lanfear, R.; Frandsen, P.B.; Wright, A.M.; Senfeld, T.; Calcott, B. PartitionFinder 2: New methods for selecting partitioned models of evolution for molecular and morphological phylogenetic analyses. Mol. Biol. Evol. 2016, 34, 772-773. [CrossRef]

45. Nguyen, L.-T.; Schmidt, H.A.; Haeseler, A.V.; Minh, B.Q. IQ-TREE: A fast and effective stochastic algorithm for estimating maximum-likelihood phylogenies. Mol. Biol. Evol. 2014, 32, 268-274. [CrossRef]

46. Ronquist, F.; Teslenko, M.; Van Der Mark, P.; Ayres, D.L.; Darling, A.; Höhna, S.; Larget, B.; Liu, L.; Suchard, M.A.; Huelsenbeck, J.P. MrBayes 3.2: Efficient Bayesian phylogenetic inference and model choice across a large model space. Syst. Biol. 2012, 61, 539-542. [CrossRef]

47. Wang, J.J.; Li, D.F.; Li, H.; Yang, M.F.; Dai, R.H. Structural and phylogenetic implications of the complete mitochondrial genome of Ledra auditura. Sci. Rep. 2019, 9, 15746. [CrossRef]

48. Ojala, D.; Montoya, J.; Attardi, G. tRNA punctuation model of RNA processing in human mitochondria. Nature 1981, 290 , 470-474. [CrossRef] [PubMed]

49. Zhang, K.J.; Zhu, W.C.; Rong, X.; Ding, X.L.; Zhang, Y.K.; Liu, J.; Chen, D.S.; Du, Y.; Hong, X.Y. The complete mitochondrial genomes of two rice planthoppers, Nilaparvata lugens and Laodelphax striatellus: Conserved genome rearrangement in Delphacidae and discovery of new characteristics of atp8 and tRNA genes. BMC Genom. 2013, 14, 417. [CrossRef] [PubMed]

50. Doublet, V.; Ubrig, E.; Alioua, A.; Bouchon, D.; Marcadé, I.; Maréchal-Drouard, L. Large gene overlaps and tRNA processing in the compact mitochondrial genome of the crustacean Armadillidium vulgare. RNA Biol. 2015, 12, 1159-1168. [CrossRef]

51. Cameron, S.L.; Whiting, M.F. The complete mitochondrial genome of the tobacco hornworm, Manduca sexta, (Insecta: Lepidoptera: Sphingidae), and an examination of mitochondrial gene variability within butterflies and moths. Gene 2008, 408, 112-123. [CrossRef] [PubMed]

52. Sheffield, N.C.; Song, H.; Cameron, S.L.; Whiting, M.F. A comparative analysis of mitochondrial genomes in Coleoptera (Arthropoda: Insecta) and genome descriptions of six new beetles. Mol. Biol. Evol. 2008, 25, 2499-2509. [CrossRef]

53. Zhang, H.; Zhang, Y.L.; Duan, Y.N. DNA barcoding of Deltocephalus Burmeister leafhoppers (Cicadellidae, Deltocephalinae, Deltocephalini) in China. Zookeys 2019, 867, 55-71. [CrossRef]

54. Huang, W.J.; Zhang, Y.L. Two new species in the genus Destinoides (Hemiptera: Cicadellidae, Ledrinae) from China, with DNA barcoding data. Zootaxa 2019, 4711, 571-578. [CrossRef]

55. Wang, Y.; Nansen, C.; Zhang, Y.L. Integrative insect taxonomy based on morphology, mitochondrial DNA, and hyperspectral reflectance profiling. Zool. J. Linn. Soc. 2016, 177, 378-394. [CrossRef]

56. Ahmed, M. Biotaxonomy of typhlocybine leafhoppers of Pakistan. In Proceedings of the 1st International Workshop on Leafhoppers and Planthoppers (Auchenorrhyncha) of Economic Importance, London, UK, 4-7 October 1982; Commonwealth Institute of Entomology: London, UK, 1983; pp. 179-183.

57. Chen, X.X.; Li, C.; Song, Y.H. The complete mitochondrial genomes of two erythroneurine leafhoppers (Hemiptera, Cicadellidae, Typhlocybinae, Erythroneurini) with assessment of the phylogenetic status and relationships of tribes of Typhlocybinae. Zookeys 2021, 1037, 137-159. [CrossRef]

58. Young, D.A. A reclassification of Western Hemisphere Typhlocybinae (Homoptera, Cicadellidae). Univ. Kans. Sci. Bull. 1952, 35, 1-217. [CrossRef]

59. Ruppel, R.F. A summary of the tribes proposed in Typhlocybinae (Hemiptera, Cicadellidae). Mich. Acad. 1987, $19,29-35$. 
60. Jiang, J.; Chen, X.X.; Li, C.; Song, Y.H. Mitogenome and phylogenetic analysis of typhlocybine leafoppers (Hemiptera: Cicadellidae). Sci. Rep. 2021, 11, 10053. [CrossRef]

61. Xu, Y.; Dietrich, C.H.; Zhang, Y.L.; Dmitriev, D.A.; Zhang, L.; Wang, Y.M.; Lu, S.H.; Qin, D.Z. Phylogeny of the tribe Empoascini (Hemiptera: Cicadellidae: Typhlocybinae) based on morphological characteristics, with reclassifification of the Empoasca generic group. Syst. Entomol. 2021, 46, 266-286. [CrossRef]

62. Anufriev, G.A. Les cicadellidaes de le Territoire Maritime. Horae Soc. Entomol. Unionis Sov. 1978, 60, 1-215. 